\title{
Short Codes for Quantum Channels with One Prevalent Pauli Error Type
}

\author{
Marco Chiani, Fellow, IEEE, and Lorenzo Valentini
}

\begin{abstract}
One of the main problems in quantum information systems is the presence of errors due to noise, and for this reason quantum error-correcting codes (QECCs) play a key role. While most of the known codes are designed for correcting generic errors, i.e., errors represented by arbitrary combinations of Pauli $X, Y$ and $Z$ operators, in this paper we investigate the design of stabilizer QECC able to correct a given number $e_{\mathrm{g}}$ of generic Pauli errors, plus $e_{\mathrm{Z}}$ Pauli errors of a specified type, e.g., $Z$ errors. These codes can be of interest when the quantum channel is asymmetric in that some types of error occur more frequently than others. We first derive a generalized quantum Hamming bound for such codes, then propose a design methodology based on syndrome assignments. For example, we found a $[[9,1]]$ quantum error-correcting code able to correct up to one generic qubit error plus one $Z$ error in arbitrary positions. This, according to the generalized quantum Hamming bound, is the shortest code with the specified error correction capability. Finally, we evaluate analytically the performance of the new codes over asymmetric channels.
\end{abstract}

Index Terms-Quantum Information, Quantum Error Correction, Quantum Hamming Bound, Asymmetric Quantum Channels.

\section{INTRODUCTION}

The possibility to exploit the unique features of quantum mechanics is paving the way to new approaches for acquiring, processing and transmitting information [1]-[5]. In this regard, one of the main problems is the noise caused by unwanted interaction of the quantum information with the environment. Error correction techniques are therefore essential for quantum computation, quantum memories and quantum communication systems [6]-[9]. Compared to the classical case, quantum error correction is made more difficult by the laws of quantum mechanics which imply that qubits cannot be copied or measured without perturbing state superposition [10]. Moreover, there is continuum of errors that could occur on a qubit. However, it has been shown that in order to correct an arbitrary qubit error it is sufficient to consider error correction on the discrete set of Pauli operators, i.e., the bit flip $\boldsymbol{X}$, phase flip $\boldsymbol{Z}$, and combined bit-phase flip $\boldsymbol{Y}[\overline{6}],[11]-[13]$. Hence, we can consider in general a channel introducing qubit errors $\boldsymbol{X}, \boldsymbol{Y}$, and $\boldsymbol{Z}$ with

Manuscript received February 11, 2020; revised June 1, 2020; accepted July 20, 2020. Date of publication MONTH DD, 2020; date of current version MONTH DD, 2020. This work was supported in part by the Italian Ministry for Education, University and Research under the program Dipartimenti di Eccellenza (2018-2022). This paper was presented in part at the Quantum Computing Workshop, 2020 International Conference on Computational Science (ICCS).

The authors are with the Department of Electrical, Electronic, and Information Engineering "Guglielmo Marconi" and CNIT, University of Bologna, 40136 Bologna, Italy (e-mail: \{marco.chiani, lorenzo.valentini13\}@unibo.it).

Digital Object Identifier 10.1109/JSAIT.2020.XXXXXXX probabilities $p_{\mathrm{X}}, p_{\mathrm{Y}}$, and $p_{\mathrm{Z}}$, respectively, and leaving the qubit intact with probability $1-\rho$, where $\rho=p_{\mathrm{X}}+p_{\mathrm{Y}}+p_{\mathrm{Z}}$. A special case of this model is the so-called depolarizing channel for which $p_{\mathrm{X}}=p_{\mathrm{Y}}=p_{\mathrm{Z}}=\rho / 3$. Quantum error-correcting codes for this channel are thus naturally designed to protect against equiprobable Pauli errors [14]-[16].

However, not all channels exhibit this symmetric behaviour of Pauli errors as, in some situations, some types of error are more likely than others [17]. In fact, depending on the technology adopted for the system implementation, the different types of Pauli error can have quite different probabilities of occurrence, leading to asymmetric quantum channels [18][20].

Owing to this considerations, it can be useful to investigate the design of quantum codes with error correction capabilities tailored to specific channel models. For example, codes for the amplitude damping channel have been proposed in [21][26], while quantum error-correcting codes for more general asymmetric channels are investigated in [17]-[19], [27]. In particular, asymmetric Calderbank Shor Steane (CSS) codes, where the two constituent classical parity check matrices are chosen with different error correction capability (e.g., Bose Chaudhuri Hocquenghem $(\mathrm{BCH})$ codes for $\boldsymbol{X}$ errors and low density parity check (LDPC) codes for $\boldsymbol{Z}$ errors), are investigated in [17], [18]. Inherent to the CSS construction there are two distinct error correction capabilities for the $\boldsymbol{X}$ and the $Z$ errors; the resulting asymmetric codes, denoted as $\left[\left[n, k, d_{\mathrm{X}} / d_{\mathrm{Z}}\right]\right]$, can correct up to $t_{\mathrm{X}}=\left\lfloor\left(d_{\mathrm{X}}-1\right) / 2\right\rfloor$ Pauli $\boldsymbol{X}$ errors and $t_{\mathrm{Z}}=\left\lfloor\left(d_{\mathrm{Z}}-1\right) / 2\right\rfloor$ Pauli $\boldsymbol{Z}$ errors per codeword. Due to the possibility of employing tools from classical error correction, many works have been focused on asymmetric codes based on the CSS construction, which, however, may not lead to the shortest codes (e.g., for the symmetric channel compare the $[[7,1]]$ CSS code with the shortest $[[5,1]]$ code [15], [16]).

In this paper we consider the Pauli-twirled asymmetric channel associated to the combination of amplitude damping and dephasing channels [18]. This model has $p_{\mathrm{X}}=p_{\mathrm{Y}}$ and $p_{\mathrm{Z}}=A \rho /(A+2)$, where $\rho$ is the error probability, and the asymmetry is accounted for by the parameter $A=p_{\mathrm{Z}} / p_{\mathrm{X}}$. This parameter is a function of the relaxation time, $T_{1}$, and the dephasing time, $T_{2}$, which are in general different, leading to $A>1$ [19], [27]. For this channel we study stabilizer codes able to correct a given number $e_{\mathrm{g}}$ of generic Pauli errors, plus a number $e_{Z}$ of Pauli errors of a specified type (e.g., $Z$ errors). We denote these as asymmetric $[[n, k]]$ codes with correction capability $\left(e_{\mathrm{g}}, e_{\mathrm{Z}}\right)$. Since we are targeting the shortest codes we do not constrain the design to CSS codes.

Specifically, we first derive a generalized version of the 
quantum Hamming bound, which was originally developed for codes able to correct up to a number $t$ of generic errors. The new generalized bound is valid also for codes with asymmetric error correction capability $\left(e_{\mathrm{g}}, e_{\mathrm{Z}}\right)$. Then, we construct, by a procedure based on syndrome assignment, a $[[9,1]]$ code with $\left(e_{\mathrm{g}}=1, e_{\mathrm{Z}}=1\right)$ which, according to the new quantum Hamming bound, is the shortest possible code. We extend the construction method to the class of $[[n, 1]]$ codes with $e_{\mathrm{g}}=1$ and arbitrary $e_{\mathrm{Z}}$, and provide as an example a $[[13,1]]$ code with $\left(e_{\mathrm{g}}=1, e_{\mathrm{Z}}=2\right)$. Finally, we analytically compare the error correction capability of the new and of previously known codes, over asymmetric channels.

\section{A. Notation}

Throughout the paper we will use the following notation. A qubit is an element of the two-dimensional Hilbert space $\mathcal{H}^{2}$, with basis $|0\rangle$ and $|1\rangle[12$. An $n$-tuple of qubits ( $n$ qubits) is an element of the $2^{n}$-dimensional Hilbert space, $\mathcal{H}^{2^{n}}$, with basis composed by all possible tensor products $\left|i_{1}\right\rangle\left|i_{2}\right\rangle \cdots\left|i_{n}\right\rangle$, with $i_{j} \in\{0,1\}, 1 \leq j \leq n$. The Pauli operators, denoted as $\boldsymbol{I}, \boldsymbol{X}, \boldsymbol{Z}$, and $\boldsymbol{Y}$, are defined by $\boldsymbol{I}|a\rangle=|a\rangle, \boldsymbol{X}|a\rangle=$ $|a \oplus 1\rangle, \boldsymbol{Z}|a\rangle=(-1)^{a}|a\rangle$, and $\boldsymbol{Y}|a\rangle=i(-1)^{a}|a \oplus 1\rangle$ for $a \in\{0,1\}$. These operators either commute or anticommute. With $[[n, k]]$ we indicate a QECC that encodes $k$ data qubits $|\varphi\rangle$ into a codeword of $n$ qubits $|\psi\rangle$. We use the stabilizer formalism, where a stabilizer code $\mathcal{C}$ is generated by $n-k$ independent and commuting operators $\boldsymbol{G}_{i} \in \mathcal{G}_{n}$, called generators [12], [28], [29]. The code $\mathcal{C}$ is the set of quantum states $|\psi\rangle$ satisfying $\boldsymbol{G}_{i}|\psi\rangle=|\psi\rangle, i=1,2, \ldots, n-k$. Assume a codeword $|\psi\rangle \in \mathcal{C}$ affected by a channel error described by the operator $\boldsymbol{E} \in \mathcal{G}_{n}$. For error correction, the received state $\boldsymbol{E}|\psi\rangle$ is measured according to the generators $\boldsymbol{G}_{1}, \boldsymbol{G}_{2}, \ldots, \boldsymbol{G}_{n-k}$, resulting in a quantum error syndrome $\boldsymbol{s}(\boldsymbol{E})=\left(s_{1}, s_{2}, \ldots, s_{n-k}\right)$, with each $s_{i}=0$ or 1 depending on the fact that $\boldsymbol{E}$ commutes or anticommutes with $\boldsymbol{G}_{i}$, respectively. Note that the syndrome depends on $\boldsymbol{E}$ and not on the particular q-codeword $|\psi\rangle$. Moreover, measuring the syndrome does not change the quantum state, which remains $\boldsymbol{E}|\psi\rangle$. Let $\mathcal{S}=\left\{\boldsymbol{s}^{(1)}, \boldsymbol{s}^{(2)}, \ldots, \boldsymbol{s}^{(m)}\right\}$ be the set of $m=2^{n-k}$ possible syndromes, with $\boldsymbol{s}^{(1)}=(0,0, \ldots, 0)$ denoting the syndrome of the operators $\boldsymbol{E}$ (including the identity $\boldsymbol{I}$, i.e., the no-errors operator) such that $\boldsymbol{E}|\psi\rangle$ is still a valid qcodeword. A generic Pauli error $\boldsymbol{E} \in \mathcal{G}_{n}$ can be described by specifying the single Pauli errors on each qubit. We will use when necessary $\boldsymbol{X}_{i}, \boldsymbol{Y}_{i}$, and $\boldsymbol{Z}_{i}$ to denote the Pauli error $\boldsymbol{X}, \boldsymbol{Y}$, and $\boldsymbol{Z}$, respectively, on the $i$-th qubit of a codeword.

\section{HAMming Bounds FOR QUANTUM ASYMMETRIC CODES}

The standard quantum Hamming bound (QHB) gives a necessary condition for the existence of non-degenerate codes able to correct generic errors. It states that a QECC which encodes $k$ qubits in $n$ qubits can correct up to $t$ generic errors per codeword only if [12], [30]

$$
2^{n-k} \geq \sum_{j=0}^{t}\left(\begin{array}{l}
n \\
j
\end{array}\right) 3^{j}
$$

The bound is easily proved by noticing that the number of syndromes, $2^{n-k}$, must be at least equal to that of the distinct errors we want to correct. Since for each position there could be three Pauli errors $(\boldsymbol{X}, \boldsymbol{Y}$ or $\boldsymbol{Z})$, the number of distinct patterns having $j$ qubits in error is $\left(\begin{array}{c}n \\ j\end{array}\right) 3^{j}$, and this gives the bound (1).

In this paper we investigate non-degenerate QECCs which can correct some generic errors $(\boldsymbol{X}, \boldsymbol{Y}$ or $\boldsymbol{Z})$, plus some fixed Pauli type errors (e.g., $Z$ errors). We derive therefore the following generalized quantum Hamming bound (GQHB).

Theorem 1 (Generalized Quantum Hamming Bound). A quantum code which encodes $k$ qubits in $n$ qubits can correct up to $e_{\mathrm{g}}$ generic errors plus up to $e_{\mathrm{Z}}$ fixed Pauli type errors (e.g., $Z$ errors) per codeword only if

$$
2^{n-k} \geq \sum_{j=0}^{e_{\mathrm{g}}+e_{\mathrm{Z}}}\left(\begin{array}{l}
n \\
j
\end{array}\right) \sum_{i=0}^{e_{\mathrm{g}}}\left(\begin{array}{l}
j \\
i
\end{array}\right) 2^{i}
$$

Proof. For the proof we need to enumerate the different patterns of error. The number of patterns of up to $e_{\mathrm{g}}$ generic errors is given by (1) with $t=e_{\mathrm{g}}$. Then, we have to add the number of configurations with $e_{\mathrm{g}}<j \leq e_{\mathrm{g}}+e_{\mathrm{Z}}$ errors, composed by $e_{\mathrm{g}}$ generic Pauli errors and the remaining $j-e_{\mathrm{g}}$ Pauli $Z$ errors. We can write

$$
2^{n-k} \geq \sum_{j=0}^{e_{\mathrm{g}}}\left(\begin{array}{l}
n \\
j
\end{array}\right) 3^{j}+\sum_{j=e_{\mathrm{g}}+1}^{e_{\mathrm{g}}+e_{\mathrm{z}}}\left(\begin{array}{l}
n \\
j
\end{array}\right)\left[3^{j}-f\left(j ; e_{\mathrm{g}}\right)\right]
$$

where $f\left(j ; e_{\mathrm{g}}\right)$ is a function that returns the number of noncorrectable patterns of $j$ errors. This is the solution of the following combinatorial problem: given $j$ positions of the errors, count the number of all combinations with more than $e_{\mathrm{g}}$ symbols from the set $\mathcal{P}_{\mathrm{XY}}=\{\boldsymbol{X}, \boldsymbol{Y}\}$ and the remaining from the set $\mathcal{P}_{\mathrm{Z}}=\{\boldsymbol{Z}\}$. We have therefore

$$
f\left(j ; e_{\mathrm{g}}\right)=\sum_{i=0}^{j-e_{\mathrm{g}}-1}\left(\begin{array}{l}
j \\
i
\end{array}\right) 2^{j-i}
$$

which allows to write

$$
\begin{aligned}
g\left(j ; e_{\mathrm{g}}\right) & =3^{j}-f\left(j ; e_{\mathrm{g}}\right) \\
& =\sum_{i=0}^{j}\left(\begin{array}{l}
j \\
i
\end{array}\right) 2^{j-i}-\sum_{i=0}^{j-e_{\mathrm{g}}-1}\left(\begin{array}{l}
j \\
i
\end{array}\right) 2^{j-i} \\
& =\sum_{i=j-e_{\mathrm{g}}}^{j}\left(\begin{array}{l}
j \\
i
\end{array}\right) 2^{j-i}=\sum_{i=0}^{e_{\mathrm{g}}}\left(\begin{array}{l}
j \\
i
\end{array}\right) 2^{i} .
\end{aligned}
$$

It is easy to see that $g\left(j ; e_{\mathrm{g}}\right)$ is equal to $3^{j}$ if $j \leq e_{\mathrm{g}}$, so substituting and incorporating the summation in (3) we finally obtain

$$
2^{n-k} \geq \sum_{j=0}^{e_{\mathrm{g}}+e_{\mathrm{Z}}}\left(\begin{array}{l}
n \\
j
\end{array}\right) g\left(j ; e_{\mathrm{g}}\right)=\sum_{j=0}^{e_{\mathrm{g}}+e_{\mathrm{Z}}}\left(\begin{array}{l}
n \\
j
\end{array}\right) \sum_{i=0}^{e_{\mathrm{g}}}\left(\begin{array}{l}
j \\
i
\end{array}\right) 2^{i}
$$

The GQHB in (2) can be used to compare codes which can correct $t$ generic errors with codes correcting a total of $t$ errors, with $e_{\mathrm{g}}$ of them generic and the others $t-e_{\mathrm{g}}$ of a fixed type. In Table 1 we report the minimum code lengths 


\begin{tabular}{lcccc}
\hline & $t=1$ & $t=2$ & $t=3$ & $t=4$ \\
\hline$k=1$ & 5,5 & 10,9 & 15,12 & 20,15 \\
$k=2$ & 7,7 & 12,10 & 16,14 & 21,17 \\
$k=3$ & 8,8 & 13,12 & 18,15 & 23,19 \\
\hline
\end{tabular}

TABLE I: Comparison between the minimum code lengths $n_{\mathrm{min}}^{\mathrm{QHB}}, n_{\mathrm{min}}^{\mathrm{GQHB}}$ according to the Hamming bounds (1) and (2), respectively. For the GQHB the bounds refer to $t=e_{\mathrm{g}}+e_{\mathrm{Z}}$ with $e_{\mathrm{g}}=1$.

$n_{\text {min }}$ resulting from the Hamming bounds, for different values of the total number of errors $t$, and assuming $e_{\mathrm{g}}=1$ for the GQHB. From the table we can observe the possible gain in qubits for the asymmetric case.

\section{CONSTRUCTION OF SHORT ASYMMETRIC CODES BY SYNDROME ASSIGNMENT}

In this section we present a construction of short stabilizer asymmetric codes with $k=1$ and $e_{\mathrm{g}}=1$, i.e., for $[[n, 1]]$ QECCs with error correction capability $\left(1, e_{\mathrm{Z}}\right)$. The design is based on the error syndromes: specifically, we proceed by assigning different syndromes to the different correctable error patters.

Let us first observe that the vector syndrome of a composed error $\boldsymbol{E}=\boldsymbol{E}_{1} \boldsymbol{E}_{2}$, with $\boldsymbol{E}_{1}, \boldsymbol{E}_{2} \in \mathcal{G}_{n}$, can be expressed as $\boldsymbol{s}(\boldsymbol{E})=\boldsymbol{s}\left(\boldsymbol{E}_{1} \boldsymbol{E}_{2}\right)=\boldsymbol{s}\left(\boldsymbol{E}_{2} \boldsymbol{E}_{1}\right)=\boldsymbol{s}\left(\boldsymbol{E}_{1}\right) \oplus \boldsymbol{s}\left(\boldsymbol{E}_{2}\right)$ where $\oplus$ is the elementwise modulo 2 addition. Moreover, $\boldsymbol{X} \boldsymbol{Z}=i \boldsymbol{Y}$, and for the syndromes we have $\boldsymbol{s}\left(\boldsymbol{X}_{i} \boldsymbol{Z}_{i}\right)=\boldsymbol{s}\left(\boldsymbol{Y}_{i}\right)$, $\boldsymbol{s}\left(\boldsymbol{X}_{i} \boldsymbol{Y}_{i}\right)=\boldsymbol{s}\left(\boldsymbol{Z}_{i}\right)$, and $\boldsymbol{s}\left(\boldsymbol{Y}_{i} \boldsymbol{Z}_{i}\right)=\boldsymbol{s}\left(\boldsymbol{X}_{i}\right)$. Hence, once we have assigned the syndromes for the single error patterns $\boldsymbol{X}_{i}$ and $Z_{i}$, with $i=1, \ldots, n$, the syndromes for all possible errors are automatically determined.

In the following, if not specified otherwise, the indexes $i, j$ will run from 1 to $n$, and the index $\ell$ will run from 1 to $n-1$. By definition the weight of a syndrome is the number of non-zero elements in the associated vector.

\section{A. Construction of $[[n, 1]]$ QECCs with $e_{\mathrm{g}}=1, e_{\mathrm{Z}}=1$}

For this case we need to assign $2 n$ syndromes $\boldsymbol{s}\left(\boldsymbol{X}_{i}\right)$ and $\boldsymbol{s}\left(\boldsymbol{Z}_{i}\right)$ such that the syndromes of the errors $\boldsymbol{I}, \boldsymbol{X}_{i}, \boldsymbol{Y}_{i}, \boldsymbol{Z}_{i}$, $\boldsymbol{X}_{i} \boldsymbol{Z}_{j}, \boldsymbol{Y}_{i} \boldsymbol{Z}_{j}$, and $\boldsymbol{Z}_{i} \boldsymbol{Z}_{j}$, are all different $\forall i, j$ with $i \neq j$. We aim to construct the shortest possible code according to the GQHB, i.e., a code with $n=9$ (see Table I). We start by assigning the syndromes of $\boldsymbol{Z}_{i}$ as reported in the Table II In particular, we associate the all-one syndrome to a $\boldsymbol{Z}$ error on the last qubit, i.e., $\boldsymbol{s}\left(\boldsymbol{Z}_{9}\right)=(1,1, \ldots, 1)$. Therefore, $\boldsymbol{s}\left(\boldsymbol{Z}_{9}\right)$ has weight $n-k=8$. With this choice we have assigned all possible syndromes of weight 1 and 8 . Also, the combinations of $\boldsymbol{Z}_{i} \boldsymbol{Z}_{j}$ with $i \neq j$, cover all possible syndromes of weight 2 and 7 .

To assign the syndromes of $\boldsymbol{X}_{i}$ we then use a Monte Carlo approach. To reduce the search space, i.e., the set of possible syndromes, we observe the following:

- The weight of $\boldsymbol{s}\left(\boldsymbol{X}_{i}\right)$ cannot be 3 or 6 . This is because otherwise $\boldsymbol{s}\left(\boldsymbol{Z}_{j} \boldsymbol{X}_{i}\right)$ would have weight 2 or 7 for some

\begin{tabular}{ccccccccc}
\hline & $s_{8}$ & $s_{7}$ & $s_{6}$ & $s_{5}$ & $s_{4}$ & $s_{3}$ & $s_{2}$ & $s_{1}$ \\
\hline $\boldsymbol{Z}_{1}$ & 0 & 0 & 0 & 0 & 0 & 0 & 0 & 1 \\
$\boldsymbol{Z}_{2}$ & 0 & 0 & 0 & 0 & 0 & 0 & 1 & 0 \\
$\boldsymbol{Z}_{3}$ & 0 & 0 & 0 & 0 & 0 & 1 & 0 & 0 \\
$\boldsymbol{Z}_{4}$ & 0 & 0 & 0 & 0 & 1 & 0 & 0 & 0 \\
$\boldsymbol{Z}_{5}$ & 0 & 0 & 0 & 1 & 0 & 0 & 0 & 0 \\
$\boldsymbol{Z}_{6}$ & 0 & 0 & 1 & 0 & 0 & 0 & 0 & 0 \\
$\boldsymbol{Z}_{7}$ & 0 & 1 & 0 & 0 & 0 & 0 & 0 & 0 \\
$\boldsymbol{Z}_{8}$ & 1 & 0 & 0 & 0 & 0 & 0 & 0 & 0 \\
$\boldsymbol{Z}_{9}$ & 1 & 1 & 1 & 1 & 1 & 1 & 1 & 1 \\
\hline
\end{tabular}

TABLE II: Assigned syndromes for single Pauli $Z$ errors.

\begin{tabular}{lcccccccc}
\hline & $s_{8}$ & $s_{7}$ & $s_{6}$ & $s_{5}$ & $s_{4}$ & $s_{3}$ & $s_{2}$ & $s_{1}$ \\
\hline $\boldsymbol{X}_{1}$ & 1 & 0 & 1 & 1 & 1 & 0 & 0 & 0 \\
$\boldsymbol{X}_{2}$ & 1 & 0 & 0 & 1 & 0 & 1 & 0 & 1 \\
$\boldsymbol{X}_{3}$ & 0 & 0 & 1 & 0 & 1 & 0 & 1 & 1 \\
$\boldsymbol{X}_{4}$ & 1 & 1 & 1 & 0 & 0 & 1 & 0 & 0 \\
$\boldsymbol{X}_{5}$ & 0 & 1 & 0 & 0 & 1 & 1 & 0 & 1 \\
$\boldsymbol{X}_{6}$ & 1 & 1 & 0 & 0 & 0 & 0 & 1 & 1 \\
$\boldsymbol{X}_{7}$ & 0 & 0 & 1 & 1 & 0 & 1 & 1 & 0 \\
$\boldsymbol{X}_{8}$ & 0 & 1 & 0 & 1 & 1 & 0 & 1 & 0 \\
$\boldsymbol{X}_{9}$ & 1 & 0 & 0 & 0 & 1 & 1 & 1 & 0 \\
\hline
\end{tabular}

TABLE III: Syndromes for single Pauli $\boldsymbol{X}$ errors.

$i$ and $j$, which are already assigned for errors of the type $\boldsymbol{Z}_{i} \boldsymbol{Z}_{j}$. Therefore the possible weights for $\boldsymbol{s}\left(\boldsymbol{X}_{i}\right)$ are only 4 and 5 . The same observation applies to $\boldsymbol{s}\left(\boldsymbol{Y}_{i}\right)$. We then fix the weight for $s\left(\boldsymbol{X}_{i}\right)$ equal to 4 .

- We can obtain $\boldsymbol{s}\left(\boldsymbol{Y}_{\ell}\right)$ with weight 5 for $\ell=1, \ldots, 8$, by imposing to " 0 " the $\ell$-th element of the syndrome of $\boldsymbol{X}_{\ell}$. Note that $\boldsymbol{Y}_{9}$ has weight 4 since $\boldsymbol{X}_{9}$ has weight 4 .

By following the previous rules, a possible assignment obtained by Monte Carlo is reported in Table III.

From Table $\amalg$ and Table $\amalg$ we can then build the stabilizer matrix with the following procedure, where $s_{j}\left(\boldsymbol{X}_{i}\right)$ indicates the $j$-th elements of the $\boldsymbol{X}_{i}$ 's syndrome:

- if $s_{j}\left(\boldsymbol{X}_{i}\right)=0$ and $s_{j}\left(\boldsymbol{Z}_{i}\right)=0$ put the element $\boldsymbol{I}$ in position $(j, i)$ of the stabilizer matrix because it is the only Pauli operator which commutes with both.

- if $s_{j}\left(\boldsymbol{X}_{i}\right)=1$ and $s_{j}\left(\boldsymbol{Z}_{i}\right)=0$ put the element $\boldsymbol{Z}$ in position $(j, i)$ of the stabilizer matrix because it is the only Pauli operator which commutes with $Z$ and anticommute with $\boldsymbol{X}$.

- if $s_{j}\left(\boldsymbol{X}_{i}\right)=0$ and $s_{j}\left(\boldsymbol{Z}_{i}\right)=1$ put the element $\boldsymbol{X}$ in position $(j, i)$ of the stabilizer matrix because it is the only Pauli operator which commutes with $\boldsymbol{X}$ and anticommute with $Z$.

- if $s_{j}\left(\boldsymbol{X}_{i}\right)=1$ and $s_{j}\left(\boldsymbol{Z}_{i}\right)=1$ put the element $\boldsymbol{Y}$ in position $(j, i)$ of the stabilizer matrix because it is the only Pauli operator which anti-commutes with both.

The resulting stabilizer matrix, after checking the commutation conditions, is represented in Table IV] According to the GQHB the code specified in the table is therefore the shortest possible code for $k=1, e_{\mathrm{g}}=1$ and $e_{\mathrm{Z}}=1$. 


\begin{tabular}{lccccccccc}
\hline & 1 & 2 & 3 & 4 & 5 & 6 & 7 & 8 & 9 \\
\hline $\boldsymbol{G}_{1}$ & $\boldsymbol{X}$ & $\boldsymbol{Z}$ & $\boldsymbol{Z}$ & $\boldsymbol{I}$ & $\boldsymbol{Z}$ & $\boldsymbol{Z}$ & $\boldsymbol{I}$ & $\boldsymbol{I}$ & $\boldsymbol{X}$ \\
$\boldsymbol{G}_{2}$ & $\boldsymbol{I}$ & $\boldsymbol{X}$ & $\boldsymbol{Z}$ & $\boldsymbol{I}$ & $\boldsymbol{I}$ & $\boldsymbol{Z}$ & $\boldsymbol{Z}$ & $\boldsymbol{Z}$ & $\boldsymbol{Y}$ \\
$\boldsymbol{G}_{3}$ & $\boldsymbol{I}$ & $\boldsymbol{Z}$ & $\boldsymbol{X}$ & $\boldsymbol{Z}$ & $\boldsymbol{Z}$ & $\boldsymbol{I}$ & $\boldsymbol{Z}$ & $\boldsymbol{I}$ & $\boldsymbol{Y}$ \\
$\boldsymbol{G}_{4}$ & $\boldsymbol{Z}$ & $\boldsymbol{I}$ & $\boldsymbol{Z}$ & $\boldsymbol{X}$ & $\boldsymbol{Z}$ & $\boldsymbol{I}$ & $\boldsymbol{I}$ & $\boldsymbol{Z}$ & $\boldsymbol{Y}$ \\
$\boldsymbol{G}_{5}$ & $\boldsymbol{Z}$ & $\boldsymbol{Z}$ & $\boldsymbol{I}$ & $\boldsymbol{I}$ & $\boldsymbol{X}$ & $\boldsymbol{I}$ & $\boldsymbol{Z}$ & $\boldsymbol{Z}$ & $\boldsymbol{X}$ \\
$\boldsymbol{G}_{6}$ & $\boldsymbol{Z}$ & $\boldsymbol{I}$ & $\boldsymbol{Z}$ & $\boldsymbol{Z}$ & $\boldsymbol{I}$ & $\boldsymbol{X}$ & $\boldsymbol{Z}$ & $\boldsymbol{I}$ & $\boldsymbol{X}$ \\
$\boldsymbol{G}_{7}$ & $\boldsymbol{I}$ & $\boldsymbol{I}$ & $\boldsymbol{I}$ & $\boldsymbol{Z}$ & $\boldsymbol{Z}$ & $\boldsymbol{Z}$ & $\boldsymbol{X}$ & $\boldsymbol{Z}$ & $\boldsymbol{X}$ \\
$\boldsymbol{G}_{8}$ & $\boldsymbol{Z}$ & $\boldsymbol{Z}$ & $\boldsymbol{I}$ & $\boldsymbol{Z}$ & $\boldsymbol{I}$ & $\boldsymbol{Z}$ & $\boldsymbol{I}$ & $\boldsymbol{X}$ & $\boldsymbol{Y}$ \\
\hline
\end{tabular}

TABLE IV: Stabilizer for a $[[9,1]]$ QECC with $e_{\mathrm{g}}=1$ and $e_{\mathrm{Z}}=1$.

\section{B. Construction of $[[n, 1]]$ QECCs with $e_{\mathrm{g}}=1$ and $e_{\mathrm{Z}} \geqslant 1$}

The construction presented in the previous section can be generalized to the case of more fixed errors, $e_{\mathrm{Z}} \geqslant 1$. In this section we indicate $\tilde{t}=e_{\mathrm{g}}+e_{\mathrm{Z}}$. We start by adopting the same assignment proposed in Table $\amalg$ for a single $\boldsymbol{Z}$ error, i.e., $\boldsymbol{s}\left(\boldsymbol{Z}_{\ell}\right)$ has a 1 in position $\ell, \ell \neq n$, and $s\left(\boldsymbol{Z}_{n}\right)=(1,1, \ldots, 1)$. Note that $s\left(\boldsymbol{Z}_{n}\right)$ has weight $n-k=n-1$. In this way, it is easy to see that we use all possible syndromes with weight in the range $[0, \tilde{t}]$ and $[n-\tilde{t}, n-1]$, covering all possible error operators with up to $\tilde{t}$ errors of type $\boldsymbol{Z}$. For the assignment of the syndromes $\boldsymbol{s}\left(\boldsymbol{X}_{i}\right)$ we can generalize the previously exposed arguments, as follows:

- The weight of $\boldsymbol{s}\left(\boldsymbol{X}_{i}\right)$ cannot be less than $2 \tilde{t}$ or greater than $n-2 \tilde{t}$. This is because otherwise $\boldsymbol{s}\left(\boldsymbol{Z}_{j_{1}} \ldots \boldsymbol{Z}_{j_{L}} \boldsymbol{X}_{i}\right)$ would have weight in the range $[0, \tilde{t}]$ or $[n-\tilde{t}, n-$ 1] for some $L \leq e_{\mathrm{Z}}$ and some choices of $j_{1}, \ldots, j_{\mathrm{L}}$. These syndromes are already assigned for errors of the type $\boldsymbol{Z}_{j_{1}} \ldots \boldsymbol{Z}_{j_{M}}$ for some $M \leq e_{\mathrm{Z}}$ and some choices of $j_{1}, \ldots, j_{\mathrm{M}}$. Therefore the possible weights for $\boldsymbol{s}\left(\boldsymbol{X}_{i}\right)$ are in the range $[2 \tilde{t}, n-2 \tilde{t}]$. The same observation applies to $s\left(\boldsymbol{Y}_{i}\right)$.

- Setting the $\ell$-th element of the syndrome of $\boldsymbol{X}_{\ell}$ to "0" we obtain that $s\left(\boldsymbol{Y}_{\ell}\right)$ has the weight of $s\left(\boldsymbol{X}_{\ell}\right)$ increased by 1 , with $\ell=1, \ldots, n-1$. Hence, in order to have both $s\left(\boldsymbol{X}_{\ell}\right)$ and $s\left(\boldsymbol{Y}_{\ell}\right)$ in the permitted range, we must have $n-4 \tilde{t} \geq 1$. Note that this constraint can be stricter than the GQHB. For example, we cannot construct the $[[12,1]]$ code with $e_{\mathrm{g}}=1, e_{\mathrm{Z}}=2$. The comparision between the GQHB and the construnction bound $n-4 \tilde{t} \geq 1$ is reported in Table $\mathrm{V}$

- About $\boldsymbol{X}_{n}$ and $\boldsymbol{Y}_{n}$, we recall that $\boldsymbol{s}\left(\boldsymbol{Z}_{n}\right)=\boldsymbol{s}\left(\boldsymbol{X}_{n}\right) \oplus$ $\boldsymbol{s}\left(\boldsymbol{Y}_{n}\right)$ and that we choose $\boldsymbol{s}\left(\boldsymbol{Z}_{n}\right)=(1,1, \ldots, 1)$. Therefore, in the positions where the syndrome of $\boldsymbol{X}_{n}$ has a 1, the syndrome of $\boldsymbol{Y}_{n}$ has a 0 , and viceversa. As a consequence, the sum of the weights of $\boldsymbol{s}\left(\boldsymbol{X}_{n}\right)$ and $\boldsymbol{s}\left(\boldsymbol{Y}_{n}\right)$ is $n-1$. Then, a good choice is to assign to $s\left(\boldsymbol{X}_{n}\right)$ a weight $\lceil(n-1) / 2\rceil$ or $\lfloor(n-1) / 2\rfloor$. In this case, if $n$ is odd $\boldsymbol{s}\left(\boldsymbol{Y}_{n}\right)$ would have the same weight, which is in the correct range because $n-4 \tilde{t} \geq 0$ is guaranteed by the previous point; if $n$ is even the weights are still in the correct range because $n-4 \tilde{t} \geq 1$.

The procedure is summarized as Algorithm 1 For example, we obtained the $[[13,1]]$ QECC with $e_{\mathrm{g}}=1$ and $e_{\mathrm{Z}}=2$ reported in Table VI.

\begin{tabular}{ccccccc}
\hline$\tilde{t}$ & 1 & 2 & 3 & 4 & 5 & 6 \\
\hline GQHB & 5 & 9 & 12 & 15 & 18 & 21 \\
$1+4 \tilde{t}$ & 5 & 9 & 13 & 17 & 21 & 25 \\
\hline
\end{tabular}

TABLE V: Comparison between the minimum code lengths according to the generalized quantum Hamming bound (2) and the construction bound $n>=1+4 \tilde{t}$. The bounds refer to $\tilde{t}=e_{\mathrm{g}}+e_{\mathrm{Z}}$ with $e_{\mathrm{g}}=1$.

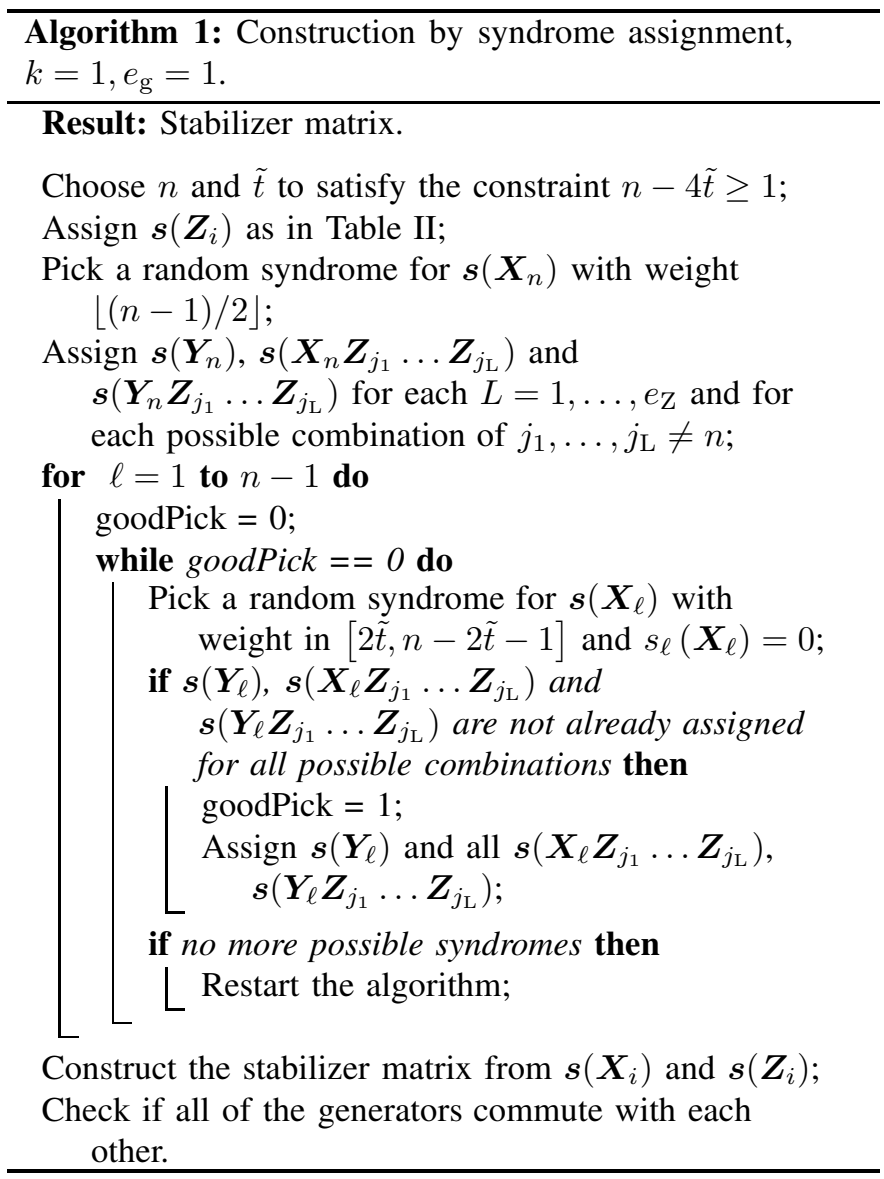

The proposed method to design asymmetric codes is based on a Monte Carlo search over a reduced syndrome assignments space. To give an idea of the time needed to find a valid code, we performed several runs of the algorithm. In our simulations the number of expected trials for the asymmetric $[[9,1]]$ case is around 20 , and for the asymmetric $[[13,1]]$ case is around 1100.

\section{Performance Analysis over Asymmetric CHANNELS}

It is well known that the Codeword Error Probability (CWEP) for a standard $[[n, k]]$ QECC which corrects up to $t$ generic errors per codeword is

$$
P_{\mathrm{e}}=1-\sum_{j=0}^{t}\left(\begin{array}{l}
n \\
j
\end{array}\right)(1-\rho)^{n-j} \rho^{j}
$$

where $\rho=p_{\mathrm{X}}+p_{\mathrm{Y}}+p_{\mathrm{Z}}$ is the qubit error probability. 


\begin{tabular}{lccccccccccccc}
\hline & 1 & 2 & 3 & 4 & 5 & 6 & 7 & 8 & 9 & 10 & 11 & 12 & 13 \\
\hline $\boldsymbol{G}_{1}$ & $\boldsymbol{X}$ & $\boldsymbol{Z}$ & $\boldsymbol{I}$ & $\boldsymbol{Z}$ & $\boldsymbol{Z}$ & $\boldsymbol{Z}$ & $\boldsymbol{I}$ & $\boldsymbol{Z}$ & $\boldsymbol{I}$ & $\boldsymbol{I}$ & $\boldsymbol{I}$ & $\boldsymbol{Z}$ & $\boldsymbol{X}$ \\
$\boldsymbol{G}_{2}$ & $\boldsymbol{I}$ & $\boldsymbol{X}$ & $\boldsymbol{Z}$ & $\boldsymbol{I}$ & $\boldsymbol{Z}$ & $\boldsymbol{Z}$ & $\boldsymbol{Z}$ & $\boldsymbol{Z}$ & $\boldsymbol{Z}$ & $\boldsymbol{I}$ & $\boldsymbol{I}$ & $\boldsymbol{I}$ & $\boldsymbol{Y}$ \\
$\boldsymbol{G}_{3}$ & $\boldsymbol{Z}$ & $\boldsymbol{Z}$ & $\boldsymbol{X}$ & $\boldsymbol{Z}$ & $\boldsymbol{I}$ & $\boldsymbol{I}$ & $\boldsymbol{I}$ & $\boldsymbol{Z}$ & $\boldsymbol{Z}$ & $\boldsymbol{Z}$ & $\boldsymbol{I}$ & $\boldsymbol{I}$ & $\boldsymbol{Y}$ \\
$\boldsymbol{G}_{4}$ & $\boldsymbol{I}$ & $\boldsymbol{I}$ & $\boldsymbol{Z}$ & $\boldsymbol{X}$ & $\boldsymbol{I}$ & $\boldsymbol{Z}$ & $\boldsymbol{I}$ & $\boldsymbol{Z}$ & $\boldsymbol{I}$ & $\boldsymbol{Z}$ & $\boldsymbol{Z}$ & $\boldsymbol{Z}$ & $\boldsymbol{Y}$ \\
$\boldsymbol{G}_{5}$ & $\boldsymbol{Z}$ & $\boldsymbol{I}$ & $\boldsymbol{Z}$ & $\boldsymbol{Z}$ & $\boldsymbol{X}$ & $\boldsymbol{I}$ & $\boldsymbol{Z}$ & $\boldsymbol{Z}$ & $\boldsymbol{I}$ & $\boldsymbol{I}$ & $\boldsymbol{Z}$ & $\boldsymbol{I}$ & $\boldsymbol{X}$ \\
$\boldsymbol{G}_{6}$ & $\boldsymbol{I}$ & $\boldsymbol{Z}$ & $\boldsymbol{I}$ & $\boldsymbol{Z}$ & $\boldsymbol{Z}$ & $\boldsymbol{X}$ & $\boldsymbol{Z}$ & $\boldsymbol{I}$ & $\boldsymbol{I}$ & $\boldsymbol{Z}$ & $\boldsymbol{Z}$ & $\boldsymbol{I}$ & $\boldsymbol{Y}$ \\
$\boldsymbol{G}_{7}$ & $\boldsymbol{I}$ & $\boldsymbol{I}$ & $\boldsymbol{Z}$ & $\boldsymbol{Z}$ & $\boldsymbol{Z}$ & $\boldsymbol{I}$ & $\boldsymbol{X}$ & $\boldsymbol{I}$ & $\boldsymbol{Z}$ & $\boldsymbol{Z}$ & $\boldsymbol{I}$ & $\boldsymbol{Z}$ & $\boldsymbol{X}$ \\
$\boldsymbol{G}_{8}$ & $\boldsymbol{Z}$ & $\boldsymbol{I}$ & $\boldsymbol{I}$ & $\boldsymbol{I}$ & $\boldsymbol{Z}$ & $\boldsymbol{Z}$ & $\boldsymbol{I}$ & $\boldsymbol{X}$ & $\boldsymbol{Z}$ & $\boldsymbol{Z}$ & $\boldsymbol{Z}$ & $\boldsymbol{I}$ & $\boldsymbol{X}$ \\
$\boldsymbol{G}_{9}$ & $\boldsymbol{Z}$ & $\boldsymbol{Z}$ & $\boldsymbol{Z}$ & $\boldsymbol{I}$ & $\boldsymbol{Z}$ & $\boldsymbol{I}$ & $\boldsymbol{I}$ & $\boldsymbol{I}$ & $\boldsymbol{X}$ & $\boldsymbol{I}$ & $\boldsymbol{Z}$ & $\boldsymbol{Z}$ & $\boldsymbol{Y}$ \\
$\boldsymbol{G}_{10}$ & $\boldsymbol{I}$ & $\boldsymbol{Z}$ & $\boldsymbol{I}$ & $\boldsymbol{I}$ & $\boldsymbol{I}$ & $\boldsymbol{I}$ & $\boldsymbol{Z}$ & $\boldsymbol{Z}$ & $\boldsymbol{Z}$ & $\boldsymbol{X}$ & $\boldsymbol{Z}$ & $\boldsymbol{Z}$ & $\boldsymbol{X}$ \\
$\boldsymbol{G}_{11}$ & $\boldsymbol{Z}$ & $\boldsymbol{I}$ & $\boldsymbol{I}$ & $\boldsymbol{Z}$ & $\boldsymbol{I}$ & $\boldsymbol{Z}$ & $\boldsymbol{Z}$ & $\boldsymbol{I}$ & $\boldsymbol{Z}$ & $\boldsymbol{I}$ & $\boldsymbol{X}$ & $\boldsymbol{Z}$ & $\boldsymbol{Y}$ \\
$\boldsymbol{G}_{12}$ & $\boldsymbol{Z}$ & $\boldsymbol{Z}$ & $\boldsymbol{Z}$ & $\boldsymbol{I}$ & $\boldsymbol{I}$ & $\boldsymbol{Z}$ & $\boldsymbol{Z}$ & $\boldsymbol{I}$ & $\boldsymbol{I}$ & $\boldsymbol{Z}$ & $\boldsymbol{I}$ & $\boldsymbol{X}$ & $\boldsymbol{X}$ \\
\hline
\end{tabular}

TABLE VI: Stabilizer for a $[[13,1]]$ QECC with $e_{\mathrm{g}}=1$ and $e_{\mathrm{Z}}=2$.

We now generalize this expression to an $[[n, k]]$ QECC which corrects up to $e_{\mathrm{g}}$ generic errors and up to $e_{\mathrm{Z}}$ Pauli $\boldsymbol{Z}$ errors per codeword, over a generic asymmetric channel with Pauli error probabilities $p_{\mathrm{X}}, p_{\mathrm{Y}}$ and $p_{\mathrm{Z}}$. To this aim, we first note that the patterns of correctable errors are those discussed in Section [II Then, by weighting each pattern with the corresponding probability of occurrence, it is not difficult to show that the performance in terms of CWEP is

$$
P_{\mathrm{e}}=1-\sum_{j=0}^{e_{\mathrm{g}}+e_{\mathrm{Z}}}\left(\begin{array}{l}
n \\
j
\end{array}\right)(1-\rho)^{n-j} \xi\left(j ; e_{\mathrm{g}}\right)
$$

where

$$
\begin{aligned}
& \xi\left(j ; e_{\mathrm{g}}\right)= \\
& \left\{\begin{array}{cc}
\rho^{j} & \text { if } j \leq e_{\mathrm{g}} \\
\sum_{i=j-e_{\mathrm{g}}}^{j}\left(\begin{array}{l}
j \\
i
\end{array}\right) p_{\mathrm{Z}}^{i} \sum_{\ell=0}^{j-i}\left(\begin{array}{c}
j-i \\
\ell
\end{array}\right) p_{\mathrm{X}}^{\ell} p_{\mathrm{Y}}^{j-i-\ell} & \text { otherwise . }
\end{array}\right.
\end{aligned}
$$

In the case of asymmetric channels with $p_{\mathrm{X}}=p_{\mathrm{Y}}=\rho /(A+$ $2), p_{\mathrm{Z}}=A \rho /(A+2)$, and $A=p_{\mathrm{Z}} / p_{\mathrm{X}}$, the expression in $(8)$ can be simplified to

$$
\begin{aligned}
P_{\mathrm{e}}= & 1-\sum_{j=0}^{e_{\mathrm{g}}+e_{\mathrm{Z}}}\left(\begin{array}{l}
n \\
j
\end{array}\right)(1-\rho)^{n-j} \rho^{j} \\
& \times\left(1-2^{j+1} \frac{(A / 2)^{j-e_{\mathrm{g}}}-1}{(A-2)(A+2)^{j}} u_{j-e_{\mathrm{g}}-1}\right)
\end{aligned}
$$

where $u_{i}=1$ if $i \geq 0$, otherwise $u_{i}=0$.

Using the previous expressions, we report in Fig. 11 the performance in terms of CWEP for different codes, assuming an asymmetric channel. The parameter $A$ accounts for the asymmetry of the channel, and for $A=1$ we have the standard depolarizing channel. In the figure we plot the CWEP for the new asymmetric $[[9,1]]$ code specified in Table IV with $e_{\mathrm{g}}=1$ and $e_{\mathrm{Z}}=1$, reported in the plot as $\mathcal{C}_{\mathrm{A}}$, over channels with asymmetry parameter $A=1,3$ and 10 . For comparison, in the same figure we report the CWEP for the known 5-qubits code, the Shor's 9-qubits code (indicated in the figure as $\mathcal{C}_{\mathrm{S}}$ ), both correcting $t=1$ generic errors, and a [[11,1]] code with $t=2$ [31]. Additionally, for a fair comparison, we analyzed the Shor's code when used in an extended mode for patterns of two errors. In fact, besides arbitrary single errors, the Shor's code can also correct some combinations of multiple errors. The most relevant multiple errors for our asymmetric channel are in the form $\boldsymbol{Z}_{i} \boldsymbol{Z}_{j}, \boldsymbol{X}_{i} \boldsymbol{Z}_{j}$, and $\boldsymbol{Y}_{i} \boldsymbol{Z}_{j}$. In this regard, it can be verified that the Shor code can be used to correct 9 out of the 36 possible $\boldsymbol{Z}_{i} \boldsymbol{Z}_{j}$ errors, all 72 possible $\boldsymbol{X}_{i} \boldsymbol{Z}_{j}$ errors, and 18 out of the 72 possible $\boldsymbol{Y}_{i} \boldsymbol{Z}_{j}$ errors. The Shor's code used with this extended error correction capability is reported in the following as $\mathcal{C}_{\mathrm{SE}}$.

About the results in Fig. 1, we remark that for symmetric codes the performance does not depend on the asymmetry parameter $A$, but just on the overall error probability $\rho$. For these codes, for a given $t$ the best CWEP is obtained with the shortest code. As expected, the performance of the new asymmetric $[[9,1]]$ code improves as $A$ increases. In particular, for the symmetric channel, $A=1$, the 5 -qubits code performs better than the new one, due to its shorter codeword size. However, already with a small channel asymmetry, $A=3$, the new code performs better than the 5-qubits code in the range of interest. For $A=10$ the new code performs similarly to the $[[11,1]]$ symmetric code with $t=2$. Asymptotically for large $A$, the channel errors tend to be of type $Z$ only, and consequently the new code behaves like a code with $t=2$. In the case of Shor code with extended error correction capability, it can be observed that when varying $A$ the curves are quite close each others. This is due that for the asymmetric channel the most important error patterns are the $\boldsymbol{Z}_{i} \boldsymbol{Z}_{j}$, and this code can correct only a subset of all possible patterns of this type. For the same reason the curve for $A=10$ is worse than that for $A=3$. We next move to the case $e_{\mathrm{g}}=1, e_{\mathrm{Z}}=2$, and compare our asymmetric $[[13,1]]$ code of Table VI with two symmetric codes, and with the [[15,1,3/7]] CSS asymmetric code [18]. The performance of our asymmetric and of symmetric codes is given in general by (8). For the CSS code, we observe that it corrects all patterns with up to $e_{\mathrm{g}}=1, e_{\mathrm{Z}}=2$ errors, plus patterns with one $\boldsymbol{X}$ and three $\boldsymbol{Z}$ errors. Therefore, the CWEP for the $[[15,1,3 / 7]]$ CSS code is

$$
\begin{aligned}
P_{\mathrm{e}}= & 1-15\left(\begin{array}{c}
14 \\
3
\end{array}\right) p_{\mathrm{X}} p_{\mathrm{Z}}^{3}(1-\rho)^{11} \\
& -\sum_{j=0}^{3}\left(\begin{array}{c}
15 \\
j
\end{array}\right)(1-\rho)^{15-j} \xi(j ; 1)
\end{aligned}
$$

where $\xi(\cdot ; \cdot)$ is given by (9). The analytical performance of 


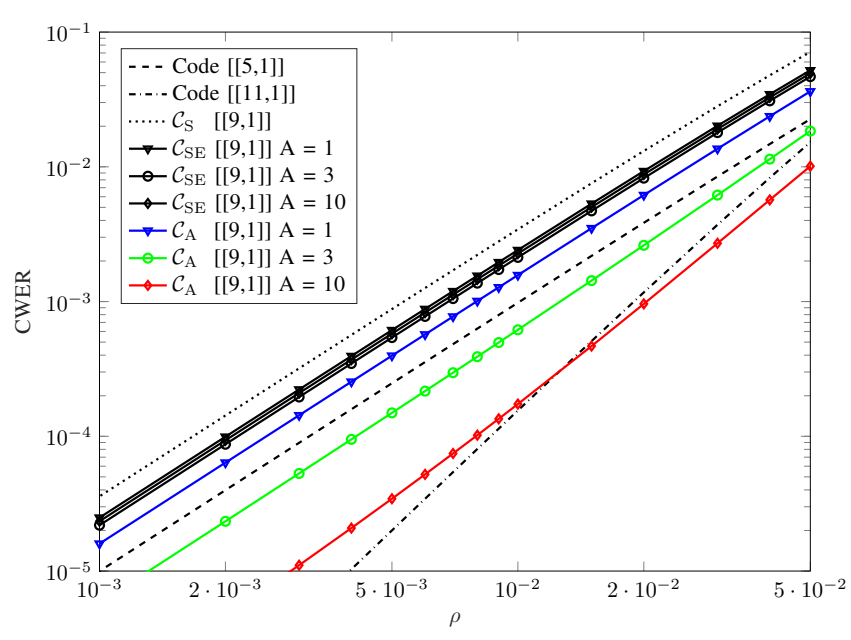

Fig. 1: Performance of short codes over an asymmetric channel, $k=1$. Code $[[5,1]]: 5$-qubits code with $t=1$. Code [[11, 1]]: 11-qubits code with $t=2 . \mathcal{C}_{\mathrm{S}}[[9,1]]: 9$-qubits code from [15] used with $t=1 . \mathcal{C}_{\mathrm{SE}}[[9,1]]: 9$-qubits code from [15] used also for the correctable two-errors patterns with at least one $\boldsymbol{Z} \cdot \mathcal{C}_{\mathrm{A}}[[9,1]]$ : 9-qubits asymmetric code with $e_{\mathrm{g}}=1, e_{\mathrm{Z}}=1$.

the different QECCs, as given by (8) and (11), is reported in Fig. 2. We can see here that the proposed $[[13,1]]$ asymmetric code performs better than the CSS, and provides a performance similar to the longer symmetric $[[17,1]]$ code for large $A$.

\section{CONCLUSIONS}

We have investigated a new class of stabilizer codes for quantum asymmetric Pauli channels, capable to correct up to $e_{\mathrm{g}}$ generic errors, plus $e_{\mathrm{Z}}$ errors of type $\boldsymbol{Z}$. For these codes we generalized the quantum Hamming bound, and derived the analytical expression of the performance over asymmetric channels. We designed a $[[9,1]]$ QECC which is the shortest, according to the new bound, capable to correct up to one generic error plus one $\boldsymbol{Z}$ error, and a [[13, 1]] QECC capable to correct up to one generic error plus two $Z$ errors. The comparison with known symmetric QECCs confirms the advantage of the proposed codes in the presence of channel asymmetry.

\section{REFERENCES}

[1] P. Zoller et al., "Quantum information processing and communication," The European Physical Journal D - Atomic, Molecular, Optical and Plasma Physics, vol. 36, no. 2, pp. 203-228, Nov 2005.

[2] H. J. Kimble, "The quantum internet," Nature, vol. 453, no. 7198, p. 1023, 2008.

[3] S. Wehner, D. Elkouss, and R. Hanson, "Quantum internet: A vision for the road ahead," Science, vol. 362, no. 6412, 2018.

[4] E. Grumbling and M. Horowitz, Eds., Quantum Computing: Progress and Prospects. Washington, DC: The National Academies Press, 2019.

[5] Quantum Networks for Open Science Workshop. Rockville, MD, USA: Office of Science US Department of Energy, 2018.

[6] E. Knill and R. Laflamme, "Theory of quantum error-correcting codes," Phys. Rev. A, vol. 55, pp. 900-911, Feb 1997.

[7] B. M. Terhal, "Quantum error correction for quantum memories," Rev. Mod. Phys., vol. 87, pp. 307-346, Apr 2015.

[8] W. Munro, A. Stephens, S. Devitt, K. Harrison, and K. Nemoto, "Quantum communication without the necessity of quantum memories," Nature Photonics, vol. 6, no. 11, p. 777, 2012.

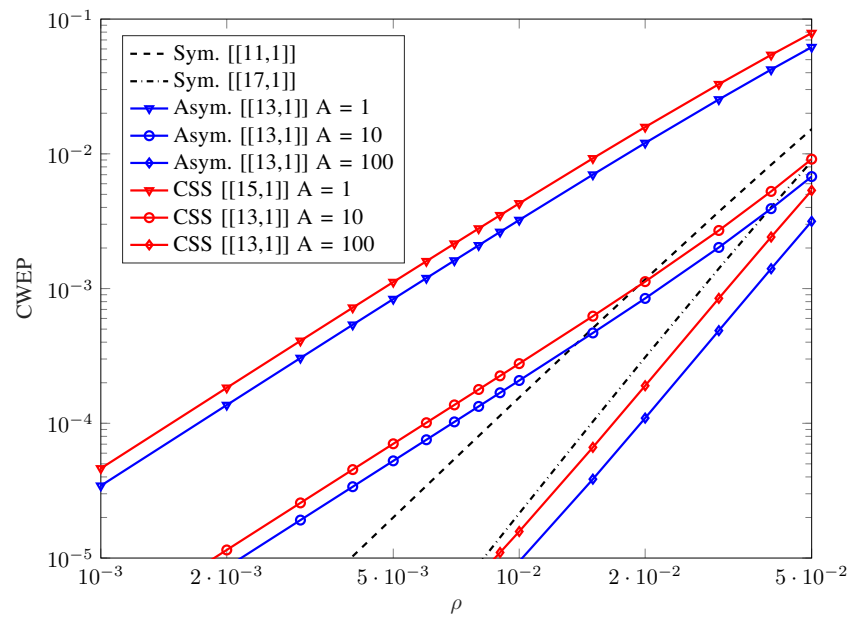

Fig. 2: Performance of short codes over an asymmetric channel, $k=1$. Symmetric codes: 11-qubits code with $t=2$ and 17 -qubits code with $t=3$. Asymmetric 13-qubits code with $e_{\mathrm{g}}=1, e_{\mathrm{Z}}=2$ and 15 -qubits code CSS with $t_{\mathrm{X}}=1$ and $t_{\mathrm{Z}}=3$.

[9] S. Muralidharan, L. Li, J. Kim, N. Lütkenhaus, M. D. Lukin, and L. Jiang, "Optimal architectures for long distance quantum communication," Scientific reports, vol. 6, p. 20463, 2016.

[10] W. K. Wootters and W. H. Zurek, "A single quantum cannot be cloned," Nature, vol. 299, no. 5886, p. 802, 1982.

[11] C. H. Bennett, D. P. DiVincenzo, J. A. Smolin, and K. W. Wootters, "Mixed state entanglement and quantum error correction," Phys. Rev. A, vol. 54, no. 5, pp. 3824-3851, 1996.

[12] M. A. Nielsen and I. L. Chuang, Quantum Computation and Quantum Information. Cambridge University Press, 2010.

[13] D. Gottesman, "An introduction to quantum error correction and faulttolerant quantum computation," arXiv preprint quant-ph/0904.2557, 2009.

[14] P. W. Shor, "Scheme for reducing decoherence in quantum computer memory," Phys. Rev. A, vol. 52, pp. R2493-R2496, Oct 1995.

[15] A. M. Steane, "Error correcting codes in quantum theory," Physical Review Letters, vol. 77, no. 5, p. 793, 1996.

[16] R. Laflamme, C. Miquel, J. P. Paz, and W. H. Zurek, "Perfect quantum error correcting code," Physical Review Letters, vol. 77, no. 1, p. 198, 1996.

[17] L. Ioffe and M. Mézard, "Asymmetric quantum error-correcting codes," Physical Review A, vol. 75, no. 3, p. 032345, 2007.

[18] P. K. Sarvepalli, A. Klappenecker, and M. Rötteler, "Asymmetric quantum codes: constructions, bounds and performance," Proceedings of the Royal Society A: Mathematical, Physical and Engineering Sciences, vol. 465, no. 2105, pp. 1645-1672, 2009.

[19] L. Gyongyosi, S. Imre, and H. V. Nguyen, "A survey on quantum channel capacities," IEEE Communications Surveys \& Tutorials, vol. 20 , no. 2, pp. 1149-1205, 2018.

[20] D. Layden, M. Chen, P. Cappellaro et al., "Efficient quantum error correction of dephasing induced by a common fluctuator," Physical review letters, vol. 124, no. 2, p. 020504, 2020.

[21] A. S. Fletcher, P. W. Shor, and M. Z. Win, "Channel-adapted quantum error correction for the amplitude damping channel," IEEE Trans. Inf. Theory, vol. 54, no. 12, pp. 5705-5718, 2008.

[22] - "Structured near-optimal channel-adapted quantum error correction," Phys. Rev. A, vol. 77, p. 012320, Jan 2008.

[23] R. Lang and P. W. Shor, "Nonadditive quantum error correcting codes adapted to the amplitude damping channel," arXiv preprint arXiv:0712.2586, 2007

[24] D. W. Leung, M. A. Nielsen, I. L. Chuang, and Y. Yamamoto, "Approximate quantum error correction can lead to better codes," Physical Review A, vol. 56, no. 4, p. 2567, 1997.

[25] P. W. Shor, G. Smith, J. A. Smolin, and B. Zeng, "High performance single-error-correcting quantum codes for amplitude damping," IEEE Trans. Inf. Theory, vol. 57, no. 10, pp. 7180-7188, 2011. 
[26] T. Jackson, M. Grassl, and B. Zeng, "Codeword stabilized quantum codes for asymmetric channels," in 2016 IEEE International Symposium on Information Theory (ISIT). IEEE, 2016, pp. 2264-2268.

[27] Z. Evans, A. Stephens, J. Cole, and L. Hollenberg, "Error correction optimisation in the presence of $\mathrm{X} / \mathrm{Z}$ asymmetry," arXiv preprint arXiv:0709.3875, 2007.

[28] D. Gottesman, "Class of quantum error-correcting codes saturating the quantum Hamming bound," Phys. Rev. A, vol. 54:1862, 1996.

[29] _ "An introduction to quantum error correction and fault-tolerant quantum computation," in Proceedings of Symposia in Applied Mathematics, vol. 68, 2009, pp. 13-58.

[30] A. Ekert and C. Macchiavello, "Quantum error correction for communication," Physical Review Letters, vol. 77, no. 12, p. 2585, 1996.

[31] M. Grassl, "Bounds on the minimum distance of linear codes and quantum codes," Online available at http://www.codetables.de. 2007, accessed on 2019-12-20.

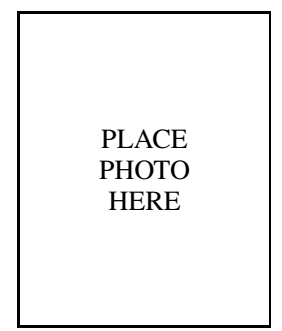

Marco Chiani (M'94-SM'02-F'11) received the Dr Ing. degree (summa cum laude) in electronic engineering and the Ph.D. degree in electronic and computer engineering from the University of Bologna, Italy, in 1989 and 1993, respectively.

$\mathrm{He}$ is a Full Professor of Telecommunications at the University of Bologna. During Summer 2001, he was a Visiting Scientist at AT\&T Research Laboratories, Middletown, NJ. Since 2003 he has been a frequent visitor at the Massachusetts Institute of Technology (MIT), Cambridge, where he presently holds a Research Affiliate appointment. His research interests are in the areas of communications theory, wireless systems, coding theory, and statistical signal processing. In 2012 he has been appointed Distinguished Visiting Fellow of the Royal Academy of Engineering, UK. He served as elected Chair (20022004) of the Radio Communications Committee of the IEEE Communication Society and as Editor (2000-2007) of Wireless Communication for the IEEE TRANSACTIONS ON COMMUNICATIONS.

He received the 2011 IEEE Communications Society Leonard G. Abraham Prize in the Field of Communications Systems, the 2012 IEEE Communications Society Fred W. Ellersick Prize, and the 2012 IEEE Communications Society Stephen O. Rice Prize in the Field of Communications Theory.

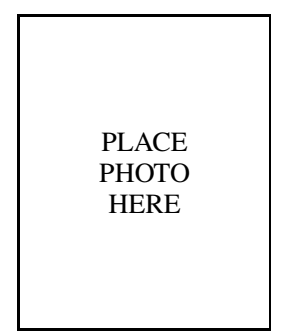

Lorenzo Valentini received the M.S. degree (summa cum laude) in Electronics and Telecommunications Engineering from the University of Bologna, Italy, in 2019. After graduation he has been with the Interdepartmental Centre for Industrial ICT Research of the University of Bologna, working on Internet of Things for the project CoACh. He is currently a Ph.D. student in Electronics, Telecommunications and Information Technologies Engineering at the University of Bologna. His research interests include communication theory, wireless sensor networks and quantum information theory. 